Planetary Systems in the Universe - Observation, Formation and Evolution

Proceedings IAU Symposium No. 202, (C)2004 IAU

Alan Penny, Pawel Artymowicz, Anne-Marie Lagrange, \& Sara Russell, eds.

\title{
Dust Rings in the Circumstellar Gas Disks
}

\author{
Taku Takeuchi and Pawel Artymowicz \\ Stockholm Observatory, S-133 36 Saltsjöbaden, Sweden
}

\begin{abstract}
In optically thin gas disks around young Vega-type stars, dust grains are exposed to the stellar radiation pressure and gas drag force. The combination of these forces pushes the grains away from the central star. Typically, $10-100 \mu \mathrm{m}$ grains migrate outward to become concentrated at the outer edge of the gas disk. A dust ring naturally forms without the help of clearing bodies, such as planets or brown dwarfs.
\end{abstract}

\section{Introduction}

Some of the young ( 10Myr) Vega-type stars, sometimes called 'transitional' or Old Pre-Main Sequence objects, have dust rings with inner holes (e.g., HR 4796A, HD 141569; Schneider et al. 1999; Augereau et al. 1999; Weinberger et al. 1999), which have been interpreted as gaps opened by planets. It is probable, indeed directly observed in some cases like HD 141569, that such young stars have low-mass gas disks (most likely remnants of primordial nebulae). In this paper, we discuss the orbital evolution of dust grains in such disks and show that a dust ring naturally forms at the outer edge of the disk (region of rapid decrease of gas density).

\section{Radial Migration of Dust Grains}

In optically thin disks, dust grains orbit around the central star with subKeplerian speed, because of the partial cancellation of gravity by the stellar radiation pressure. The ratio of radiation pressure to gravity, $\beta$, is inversely proportional to the grain size, $s$, for grains larger than a few microns. Thus, smaller grains have a lower circulation speed. The sub-micron dust is expelled by radiation from early type stars $(\beta>1)$. On the other hand, the circular velocity of gas is also mostly sub-Keplerian, because of gas pressure gradient. The ratio of pressure force to gravity, $\eta$, usually increases with radius $r$. If there is an outer edge of the gas disk (smooth cutoff spread over a few vertical scale heights), $\eta$ could be as large as $\eta \sim 0.1$ there.

Consider a grain in a gas disk. If large enough so that $\beta<\eta$, it circulates faster than the gas and experiences a head-wind. The drag force removes angular momentum from the grain, forcing it to spiral toward the star (e.g., Weidenschilling 1977). If the grain is small and $\beta>\eta$, it moves slower than the gas. The grain then gains angular momentum due to a back-wind and spirals out. The migration terminates when the grain arrives at such location where it corotates with gas $[\beta=\eta(r)]$. Grains having different values of $\beta$ (different 


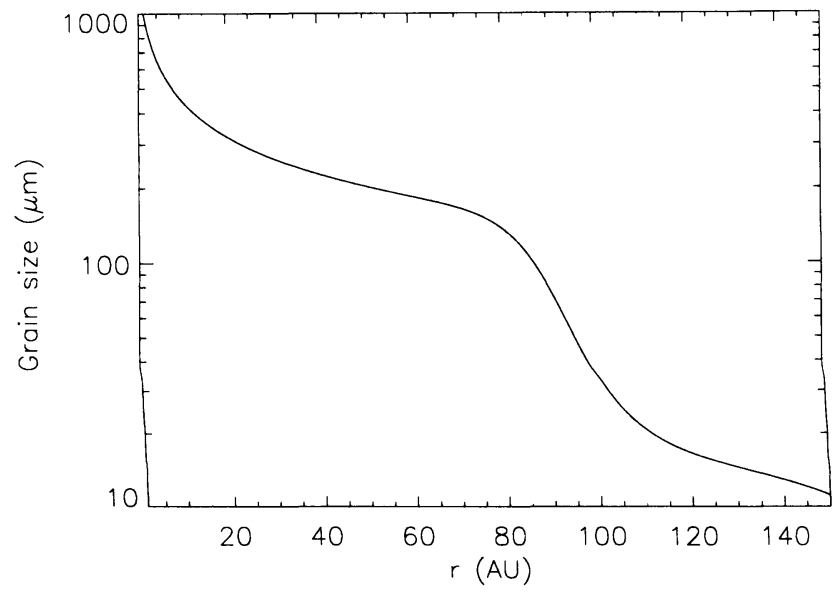

Figure 1. Size of grains terminating their migration at radius $r$, in our disk model.

size $s$ ) terminate their migration at different locations in the gas disk. Thus, the migration of dust grains tends to segregate the grains according to size.

Figure 1 shows that smaller grains migrate farther from the star than large grains. We assumed the gas to have surface density $\Sigma_{\mathrm{g}} \propto r^{-1}$ (mass $M_{\mathrm{g}}=$ $\left.10 M_{\oplus}\right)$ out to the edge at $100 \mathrm{AU}$, where $\Sigma_{\mathrm{g}}$ decreases exponentially $\left(\Sigma_{\mathrm{g}} \sim\right.$ $\exp [(r-100 \mathrm{AU}) / 5 \mathrm{AU}])$. We see that $10 \lesssim s \lesssim 100 \mu \mathrm{m}$ grains concentrate at the edge of the gas disk $(80 \lesssim r \lesssim 110 \mathrm{AU})$ where $\eta$ increases from $\sim 0.01$ to $\sim 0.1$. These grains form a dust ring at the edge of the gas disk. Grains of $1 \lesssim s \lesssim 10 \mu \mathrm{m}(0.1 \lesssim \beta<1)$ migrate far out, residing mostly outside the gas disk in an extended outer dust disk, on elliptic orbits with pericenters located at the edge of the gas disk. (Eccentricity is generated at the disk edge, and is not due to initial conditions.) As already mentioned, grains smaller than $\sim 1 \mu \mathrm{m}$ $(\beta>1)$ do not have stable orbits.

\section{Density Profiles of Dust Disks}

The disk particles are produced by collisions and/or evaporation of parent bodies such as comets, asteroids and meteorites. Suppose that parent bodies are present in the inner part of the gas disk only (say, $\sim 10 \mathrm{AU}$ ). The daughter grains migrate outward and settle in their steady orbits, from which they are later removed by collisions with other grains passing by. All the solids, including both the dust and the eroded parent meteoroids, gradually migrate outward, as they are eroded, until the fine dust is finally swept away by radiation.

We assume that the dust disk is mainly composed of grains staying in steady orbits (this assumption is appropriate if gas mass is larger than several earth masses, Takeuchi \& Artymowicz 2001). The density of grains on steady orbits is calculated by considering the balance between the supply and the removal of grains through the collisions. 


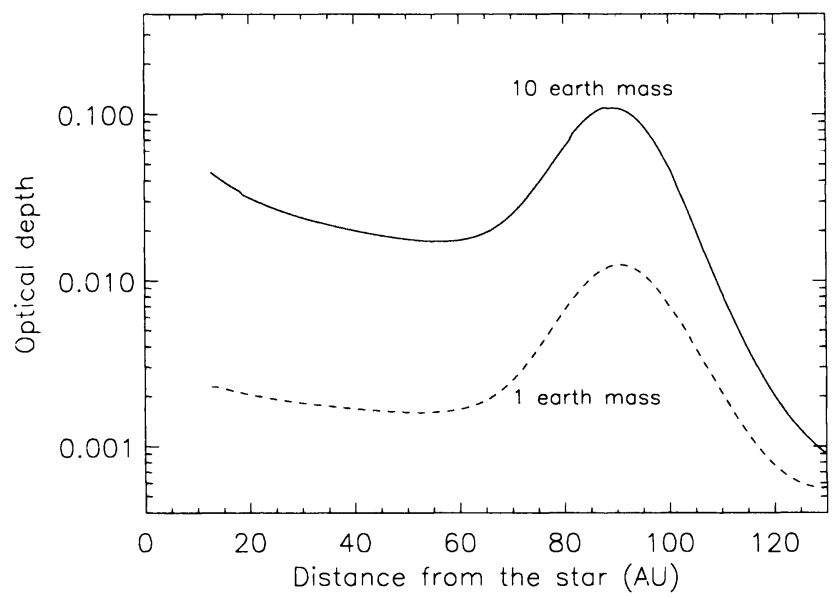

Figure 2. The vertical optical depth of dust in two models of gas disks, with $10 M_{\oplus}$ (solid line) and $1 M_{\oplus}$ (dashed line) total gas mass. The optical depth is calculated using the geometrical cross sections of grains.

The vertical optical thickness of dust is shown in Figure 2. Because the grains of $10 \lesssim s \lesssim 100 \mu \mathrm{m}$ concentrate at the edge of the gas disk ( 100AU), the dust density peaks there. The density profile of the dust disk strongly depends on the properties of the gas disk. For example, if the gas mass is smaller, the dust density is also smaller. Therefore, within our model we can derive the properties of gas disks, which are far more difficult to observe than dust disks, from the observations of dust disks.

We have shown that outer dust rings naturally form in residual gas disks of finite extent. In principle, this may be the explanation of an emerging class of dusty circumstellar rings, sometimes appearing as a single most visible feature (HR 4796A), and sometimes apparently coexisting with an inner disk/ring of dust (HD 141569).

Our models are axisymmetric, which is qualitatively consistent with observations (taking into account the anisotropic scattering by dust). The convincing proof of the presence of planetary bodies in transitional disks must include the detection of very specific, non-axisymmetric structures which can only be attributed to low-mass perturbers.

\section{References}

Augereau, J. C., Lagrange, A. M., Mouillet, D., \& Ménard, P. A. 1999, A\&A, 350, L51 Schneider, G., et al. 1999, ApJ, 513, L127

Takeuchi, T., \& Artymowicz, P. 2001, ApJ, 557, 990

Weidenschilling, S. J. 1977, MNRAS, 180, 57

Weinberger, A. J., Becklin, E. E., Schneider, G., et al. 1999, ApJ, 525, L53 